\title{
Stability Analysis for Fractional-Order Linear Singular Delay Differential Systems
}

\author{
Hai Zhang, ${ }^{1,2}$ Daiyong Wu, ${ }^{2}$ Jinde Cao, ${ }^{1,3}$ and Hui Zhang ${ }^{2}$ \\ ${ }^{1}$ Department of Mathematics, Research Center for Complex Systems and Network Sciences, \\ Southeast University, Nanjing, Jiangsu 210096, China \\ ${ }^{2}$ School of Mathematics and Computation Science, Anqing Normal University, Anqing, Anhui 246133, China \\ ${ }^{3}$ Department of Mathematics, Faculty of Science, King Abdulaziz University, Jeddah 21589, Saudi Arabia
}

Correspondence should be addressed to Jinde Cao; jdcao@seu.edu.cn

Received 14 February 2014; Accepted 4 March 2014; Published 3 April 2014

Academic Editor: Guanghui Wen

Copyright (C) 2014 Hai Zhang et al. This is an open access article distributed under the Creative Commons Attribution License, which permits unrestricted use, distribution, and reproduction in any medium, provided the original work is properly cited.

We investigate the delay-independently asymptotic stability of fractional-order linear singular delay differential systems. Based on the algebraic approach, the sufficient conditions are presented to ensure the asymptotic stability for any delay parameter. By applying the stability criteria, one can avoid solving the roots of transcendental equations. An example is also provided to illustrate the effectiveness and applicability of the theoretical results.

\section{Introduction}

Singular systems play important roles in modelling and analyzing a lot of practical systems, such as power systems, economic systems, chemical processes, and network analysis. Depending on these fields of applications, they are also called differential-algebraic systems, descriptor systems, semistate systems, or generalized state-space systems. Over the last two decades, many results regarding integer-order singular systems have been obtained (see [1-10]). In [3-10], various stability problems of integer-order singular systems have been considered by applying the different methods, such as linear matrix inequality (LMI) method [3, 4, 6, 7], $\theta$-method and BDF method [5], Lyapunov-Krasovskii functional method $[8,9]$, and Lyapunov direct method [10].

The fractional dynamics system is a recent focus of interest to many researchers [11-32]. Many practical phenomena in the fields of economics, engineering, and physics can be represented more accurately through fractional derivative formulation. The basic theory of factional calculus can be found in the monographs of Miller and Ross [11], Podlubny [12], Kilbas et al. [13], and Diethelm [14]. Moreover, Lakshmikantham et al. [15] and Baleanu et al. [16] have elaborated the theory of fractional-order dynamics systems and the recent developments.
As we all know, stability is an important performance metric for dynamic systems. Since the fractional derivative has the nonlocal property and weakly singular kernels, the analysis of stability of fractional-order systems is more complex than that of integer-order differential systems. In recent years, there are some results on the stability of fractionalorder differential systems [18-20]. For example, Li et al. [18, 19] proposed the Mittag-Leffler stability of fractional-order systems based on fractional comparison principle [18] and Lyapunov direct method [19]. On the other hand, time delay has an important effect on the stability and performance of dynamic systems. It is worth mentioning that the notable contributions have been made to the stability of fractionalorder delay differential systems (see [21-30]). In particular, Deng et al. [24] studied Lyapunov asymptotic stability of fractional linear delay differential systems by using the final-value theorem of Laplace transform. De la Sen [25] considered Robust stability of fractional-order linear delayed dynamic systems by means of fixed point theory. In [26], Li and Zhang presented a survey on the stability of fractional-order (delay) differential equations. Kaslik and Sivasundaram [27] investigated the asymptotic stability of linear fractional-order delay differential equations by using the analytical and numerical methods. By employing Lyapunov functional method, Sadati et al. [28] and Baleanu et al. [29] established Mittag-Leffler 
stability theorem and Razumikhin stability theorem for fractional-order nonlinear delay systems, respectively.

For fractional-order linear singular systems without delay, N'Doye et al. [31] studied the stabilization problems by means of LMI method. However, to the best of our knowledge, there are very few works on the stability of fractionalorder linear singular delay differential systems as reported in the current literatures except [32], in which Zhang and Jiang investigated the finite-time stability of fractional-order singular delay differential systems in terms of the Gronwall integral inequality. Compared to the stability theory of integer-order singular dynamics systems [3-10] and fractional-order delay differential systems [21-30], the stability theory of fractionalorder singular delay systems is not yet sufficiently elaborated.

In this paper, we are interested in the delay-independent stability of the Caputo fractional-order singular delay differential system as follows:

$$
\begin{gathered}
\bar{E} D^{\alpha} x(t)=\bar{A} x(t)+\bar{B} x(t-\tau), \quad t \geq 0, \\
x(t)=\bar{\varphi}(t), \quad-\tau \leq t \leq 0
\end{gathered}
$$

and the Caputo fractional-order singular neutral delay differential system

$$
\begin{gathered}
D^{\alpha}[E x(t)-C x(t-\tau)]=A x(t)+B x(t-\tau), \quad t \geq 0, \\
x(t)=\varphi(t), \quad-\tau \leq t \leq 0,
\end{gathered}
$$

where $0<\alpha<1 ; x(t) \in \mathbb{R}^{n}$ is the state vector; $D^{\alpha} x(t)$ denotes an $\alpha$ order Caputo fractional-order derivative of $x(t)$; matrices $\bar{A}, \bar{B}, A, B, C \in \mathbb{R}^{n \times n}$ and matrices $\bar{E}, E \in \mathbb{R}^{n \times n}$ are singular with $\operatorname{rank}(\bar{E})=r<n, \operatorname{rank}(E)=r<n ; \tau \in \mathbb{R}^{+}$is the time delay; and $\bar{\varphi}, \varphi$ are both the consistent initial functions.

Different from the methods in [18-32], we apply the algebraic approach to establish the delay-independently asymptotic stability criteria for system (1) and system (2). The novelty of this paper lies in the following aspects. Firstly, we synchronously take into account the factors of such systems including the Caputo's fractional-order derivative, pseudostate delay, and singular coefficient matrices. Secondly, the algebraic approach is applied to derive the sufficient conditions of the delay-independent stability, which ensure the asymptotic stability for any delay parameter $\tau \in \mathbb{R}^{+}$. Thirdly, by applying these stability criteria, one can avoid solving the roots of transcendental equations.

This paper is organized as follows. In Section 2, we introduce some definitions and preliminary facts used in the paper. In Section 3, the sufficient conditions of the delayindependently asymptotic stability for system (1) and system (2) are derived based on the algebraic approach, respectively. In Section 4, an example is provided to illustrate the effectiveness and applicability of the proposed criteria. Finally, some concluding remarks are drawn in Section 5.

\section{Preliminaries}

In this section, we recall some definitions of fractional calculus (see [11-14]) and preliminary facts used in the paper.
Definition 1 (see [12]). Riemann-Liouville's fractional integral of order $q>0$ for a function $f: \mathbb{R}^{+} \rightarrow \mathbb{R}^{n}$ is defined as

$$
D^{-q} f(t)=\frac{1}{\Gamma(q)} \int_{0}^{t}(t-s)^{q-1} f(s) d s, \quad t>0,
$$

where $\Gamma(\cdot)$ is the Gamma function.

Definition 2 (see [12]). The Riemann-Liouville's fractional derivative of order $q$ for a function $f: \mathbb{R}^{+} \rightarrow \mathbb{R}^{n}$ is defined as

$$
{ }^{\mathrm{RL}} D^{q} f(t)=\frac{1}{\Gamma(m-q)} \frac{d^{m}}{d t^{m}} \int_{0}^{t}(t-s)^{m-q-1} f(s) d s,
$$

where $0 \leq m-1 \leq q<m, m \in \mathbb{Z}^{+}$.

Definition 3 (see [12]). The Caputo's fractional derivative of order $q$ for a function $f: \mathbb{R}^{+} \rightarrow \mathbb{R}^{n}$ is defined as

$$
{ }^{C} D^{q} f(t)=\frac{1}{\Gamma(m-q)} \int_{0}^{t}(t-s)^{m-q-1} f^{(m)}(s) d s,
$$

where $0 \leq m-1 \leq q<m, m \in \mathbb{Z}^{+}$. For the sake of convenience, ${ }^{C} D^{q}$ is still written as $D^{q}$.

Definition 4 (see [12]). The Mittag-Leffler function in two parameters is defined as

$$
E_{q, \beta}(z)=\sum_{k=0}^{+\infty} \frac{z^{k}}{\Gamma(k q+\beta)},
$$

where $q>0, \beta>0$ and $z \in \mathbb{C}$ and $\mathbb{C}$ denotes the complex plane. In particular, for $\beta=1$, the Mittag-Leffler function in one parameter is defined as

$$
E_{q}(z)=\sum_{k=0}^{+\infty} \frac{z^{k}}{\Gamma(1+k q)}, \quad q>0, z \in \mathbb{C} .
$$

For $\alpha \in(0,1)$, the Laplace transform of Caputo fractional-order derivative $D^{\alpha} f(t)$ is given as follows:

$$
\mathfrak{E}\left[{ }^{c} D^{\alpha} f(t)\right]=s^{\alpha} \mathfrak{E}[f(t)]-s^{\alpha-1} f(0) .
$$

Throughout this paper, let $\mathbf{C}\left([-\tau, 0], \mathbb{R}^{n}\right)$ be the space of all continuous functions mapping the interval $[-\tau, 0]$ into $\mathbb{R}^{n}$, and $\mathbf{C}^{\mathbf{l}}\left([-\tau, 0], \mathbb{R}^{n}\right)$ denotes the space of all continuous differential functions mapping the interval $[-\tau, 0]$ into $\mathbb{R}^{n}$. The consistent initial function $\bar{\varphi}(\cdot) \in \mathbf{C}\left([-\tau, 0], \mathbb{R}^{n}\right)$ and $\varphi(\cdot) \in \mathbf{C}^{\mathbf{l}}\left([-\tau, 0], \mathbb{R}^{n}\right)$.

As discussed by Zhang [21], applying the method of steps, we obtain the following lemmas which generalize well-known results of integer-order singular systems without delay (see [1]) to fractional-order singular delay differential systems.

Definition 5. For any given two matrices $E, A \in \mathbb{R}^{n \times n}$, the matrix pair $(E, A)$ is called regular if $\operatorname{det}\left(\lambda^{\alpha} E-A\right)$ is not identically zero, where $\lambda \in \mathbb{C}$. 
Lemma 6. Assume that $(E, A)$ is regular, and $\varphi(\cdot) \in$ $\mathbf{C}^{1}\left([-\tau, 0], \mathbb{R}^{n}\right)$ is the consistent initial function, then system (2) has a unique solution on $[0,+\infty)$.

Proof. For system (2), on the interval $[-\tau, 0], x(t)=\varphi(t)$. Thus, when $t \in[0, \tau]$, system (2) is given by

$$
D^{\alpha}[E x(t)-C \varphi(t-\tau)]=A x(t)+B \varphi(t-\tau),
$$

that is,

$$
E D^{\alpha} x(t)=A x(t)+B \varphi(t-\tau)+C D^{\alpha}[\varphi(t-\tau)] .
$$

Since $(E, A)$ is regular, and $B \varphi(t-\tau)+C D^{\alpha}[\varphi(t-\tau)]$ is continuous on $[0, \tau]$, by applying Theorem 3.2 in [32], we obtain that $x_{1}(t)$ is a unique solution of system $(2)$ on $[0, \tau]$.

For $t \in[\tau, 2 \tau]$, system (2) is given by

$$
E D^{\alpha}\left[x(t)-C x_{1}(t-\tau)\right]=A x(t)+B x_{1}(t-\tau),
$$

that is,

$$
E D^{\alpha} x(t)=A x(t)+B x_{1}(t-\tau)+C D^{\alpha}\left[x_{1}(t-\tau)\right] .
$$

Since $B x_{1}(t-\tau)+C D^{\alpha}\left[x_{1}(t-\tau)\right]$ is continuous on $[\tau, 2 \tau]$, similarly, we obtain that $x_{2}(t)$ is a unique solution of system (2) on $[\tau, 2 \tau]$.

Assume that system (2) has a unique solution $x_{k}(t)$ on $[(k-1) \tau, k \tau]$. For $t \in[k \tau,(k+1) \tau]$, system $(2)$ is given by

$$
E D^{\alpha}\left[x(t)-C x_{k}(t-\tau)\right]=A x(t)+B x_{k}(t-\tau),
$$

that is,

$$
E D^{\alpha} x(t)=A x(t)+B x_{k}(t-\tau)+C D^{\alpha}\left[x_{k}(t-\tau)\right] .
$$

Since $B x_{k}(t-\tau)+C D^{\alpha}\left[x_{k}(t-\tau)\right]$ is continuous on $[k \tau,(k+1) \tau]$, similarly, we obtain that $x_{k+1}(t)$ is a unique solution of system (2) on $[k \tau,(k+1) \tau]$.

According to the mathematical induction, we know that system (2) has a unique solution on $[0,+\infty)$. Therefore, the proof is completed.

Lemma 7 (see [32]). Assume that $(\bar{E}, \bar{A})$ is regular and $\bar{\varphi}(\cdot) \epsilon$ $\mathbf{C}\left([-\tau, 0], \mathbb{R}^{n}\right)$ is the consistent initial function; then system (1) has a unique solution on $[0,+\infty)$.

Assume that the conditions in Lemmas 6 and 7 are satisfied, which ensure the existence and uniqueness of the solutions of system (1) and system (2). From Definition 3, the Caputo's fractional derivative of a constant is equal to zero; then $x(t) \equiv 0$ is the zero solution of system (1) and system (2), respectively.

Definition 8. Assume that $(\bar{E}, \bar{A})$ (or $(E, A))$ is regular; then the zero solution $x(t) \equiv 0$ of system (1) (or (2)) is called delay-independently asymptotically stable if, for any consistent initial function $\bar{\varphi}(\cdot) \in \mathbf{C}\left([-\tau, 0], \mathbb{R}^{n}\right)$ (or $\varphi(\cdot) \in$ $\left.\mathbf{C}^{\mathbf{l}}\left([-\tau, 0], \mathbb{R}^{n}\right)\right)$, its analytic solution $x(t)$ satisfies

$$
\lim _{t \rightarrow+\infty} x(t)=0
$$

for any delay parameter $\tau>0$.
Now, we introduce the first equivalent form of system (1) by means of the nonsingular transform, which is also called the standard canonical decomposition of a singular system.

Assume that $(\bar{E}, \bar{A})$ is regular, then there exist two nonsingular matrices $P, Q \in \mathbb{R}^{n \times n}$, such that system (1) is equivalent to canonical system as follows:

$$
\begin{aligned}
{ }^{c} D^{\alpha} x_{1}(t)= & \bar{A}_{1} x_{1}(t)+\bar{B}_{11} x_{1}(t-\tau) \\
& +\bar{B}_{12} x_{2}(t-\tau), \quad t \geq 0, \\
N^{c} D^{\alpha} x_{2}(t)= & x_{2}(t)+\bar{B}_{21} x_{1}(t-\tau) \\
& +\bar{B}_{22} x_{2}(t-\tau), \quad t \geq 0, \\
x_{1}(t)= & \bar{\varphi}_{1}(t), \quad-\tau \leq t \leq 0, \\
x_{2}(t)= & \bar{\varphi}_{2}(t), \quad-\tau \leq t \leq 0,
\end{aligned}
$$

where $0<\alpha<1, x_{1} \in \mathbb{R}^{r}, x_{2} \in \mathbb{R}^{n-r}$, and $N$ is nilpotent whose nilpotent index is denoted by $v=\operatorname{ind}(\bar{E}, \bar{A})$, that is, $N^{v}=0, N^{\nu-1} \neq 0$, and

$$
\begin{array}{ll}
P \bar{E} Q=\left[\begin{array}{cc}
I_{r} & 0 \\
0 & N
\end{array}\right], & P \bar{A} Q=\left[\begin{array}{cc}
\bar{A}_{1} & 0 \\
0 & I_{n-r}
\end{array}\right], \\
P \bar{B} Q=\left[\begin{array}{ll}
\bar{B}_{11} & \bar{B}_{12} \\
\bar{B}_{21} & \bar{B}_{22}
\end{array}\right], & Q^{-1} x(t)=\left[\begin{array}{l}
x_{1}(t) \\
x_{2}(t)
\end{array}\right] .
\end{array}
$$

In particular, when $\tau=0$ and ind $(\bar{E}, \bar{A})=1$, system (1) is equivalent to the following canonical system without delay:

$$
\begin{aligned}
D^{\alpha} x_{1}(t) & =\left(\bar{A}_{1}+\bar{B}_{1}\right) x_{1}(t), \quad t \geq 0, \\
0 & =x_{2}(t), \quad t \geq 0, \\
x_{1}(t) & =\bar{\varphi}_{1}(t), \quad-\tau \leq t \leq 0, \\
x_{2}(t) & =\bar{\varphi}_{2}(t), \quad-\tau \leq t \leq 0 .
\end{aligned}
$$

In addition, taking the Laplace transform on both sides of system (1) yields

$$
s^{\alpha} \bar{E} £[x(t)]-s^{\alpha-1} \bar{\varphi}(0)=\bar{A} £[x(t)]+\bar{B} £[x(t-\tau)],
$$

where $0<\alpha<1$. It follows from the properties of integral that

$$
\mathfrak{E}[x(t-\tau)]=e^{-s \tau} \mathfrak{E}[x(t)]+e^{-s \tau} \int_{-\tau}^{0} e^{-s t} \bar{\varphi}(t) d t .
$$

Thus, we obtain

$$
\begin{aligned}
& {\left[s^{\alpha} \bar{E}-\bar{A}-\bar{B} e^{-s \tau}\right] £[x(t)]} \\
& \quad=s^{\alpha-1} \bar{\varphi}(0)+\bar{B} e^{-s \tau} \int_{-\tau}^{0} e^{-s t} \bar{\varphi}(t),
\end{aligned}
$$

where

$$
\Delta(s, \tau)=\operatorname{det}\left[s^{\alpha} \bar{E}-\bar{A}-\bar{B} e^{-s \tau}\right]
$$

is the characteristic polynomial of system (1). 


\section{Stability Criteria}

In this section, we derive the sufficient conditions of the delay-independently asymptotic stability for system (1) and system (2), respectively.

Theorem 9. Assume that $(\bar{E}, \bar{A})$ is regular, and ind $(\bar{E}, \bar{A})=1$; then system (1) is delay-independently asymptotically stable if the following conditions simultaneously hold.

$\left(\mathrm{H}_{1}\right)$ All the eigenvalues $\lambda$ s of matrix $\bar{A}_{1}+\bar{B}_{1}$ satisfy

$$
\left|\arg \left(\lambda\left(\bar{A}_{1}+\bar{B}_{1}\right)\right)\right|>\frac{\alpha \pi}{2} .
$$

$\left(\mathrm{H}_{2}\right)$ For any $y \in \mathbb{R}, \tau \in \mathbb{R}^{+}$, and $i=\sqrt{-1}$, then

$$
\Delta(i y, \tau)=\operatorname{det}\left[(i y)^{\alpha} \bar{E}-\bar{A}-\bar{B} e^{-i y \tau}\right] \neq 0 .
$$

Proof. For any $\tau \in \mathbb{R}^{+}$, we only need to prove that all the roots of equation $\Delta(s, \tau)=0$ lie in open left-half complex plane.

When $\tau=0$ and $\operatorname{ind}(E, A)=1$, system (1) is reduced to the slow fractional-order subsystem

$$
D^{\alpha} x_{1}(t)=\left(\bar{A}_{1}+\bar{B}_{1}\right) x_{1}(t)
$$

and the fast subsystem

$$
0=x_{2}(t)
$$

Thus, the asymptotic stability of system (18) entirely depends on $x_{1}(t)$ irrespective of $x_{2}(t)$.

From condition $\left(\mathrm{H}_{1}\right)$, any root of equation $\operatorname{det}\left(\lambda I_{n_{1}}-\bar{A}_{1}-\right.$ $\left.\bar{B}_{1}\right)=0$ satisfies

$$
|\arg (\lambda)|>\frac{\alpha \pi}{2}
$$

Let $\lambda=s^{\alpha}$, then we have $s=\lambda^{1 / \alpha}$. It follows from inequality (27) that

$$
|\arg (s)|>\frac{\pi}{2}
$$

where $s$ is just the root of the following characteristic equation of the slow fractional-order subsystem

$$
\operatorname{det}\left(s^{\alpha} I_{r}-\bar{A}_{1}-\bar{B}_{1}\right)=0 .
$$

By computation of the determinants, we have

$$
\begin{aligned}
& \operatorname{det}\left(s^{\alpha} \bar{E}-\bar{A}-\bar{B}\right) \\
& =\operatorname{det}\left\{P^{-1}\left[\begin{array}{cc}
s^{\alpha} I_{r}-\bar{A}_{1}-\bar{B}_{1} & 0 \\
0 & -I_{n-r}
\end{array}\right] Q^{-1}\right\} \\
& =(-1)^{n-r} \operatorname{det}\left(s^{\alpha} I_{r}-\bar{A}_{1}-\bar{B}_{1}\right) \operatorname{det}\left(P^{-1} Q^{-1}\right) .
\end{aligned}
$$

Taking into account (28), (29), and (30), one can get that all the roots of equation $\operatorname{det}\left(s^{\alpha} \bar{E}-\bar{A}-\bar{B}\right)=0$ have negative real parts. Hence, it follows from $\left(\mathrm{H}_{1}\right)$ that system (18) is asymptotically stable.

On the other hand, since $\operatorname{rank}(\bar{E})=r$, the characteristic polynomial of system (1) can be expressed as

$$
\begin{aligned}
\Delta(s, \tau)= & P_{0} \cdot\left(s^{\alpha}\right)^{r}+P_{1} \cdot\left(s^{\alpha}\right)^{r-1} \\
& +\cdots+P_{r-1} \cdot s^{\alpha}+P_{r}, \quad P_{0} \neq 0
\end{aligned}
$$

where $P_{i}(i=0,1,2, \ldots, r)$ are the polynomial of the exponential function $e^{-s \tau}$, and their coefficients are composed of the elements of matrices $\bar{E}, \bar{A}, \bar{B}$.

Let $\mathfrak{R} e(s)$ represent the real part of the complex number $s$. In (31), for any $\tau \in \mathbb{R}^{+}$, if we assume that $\Re e(s) \geq 0$, then we have $\left|e^{-s \tau}\right| \leq 1$ and $P_{i}(i=0,1,2, \ldots, r)$ are bounded. Denote

$$
Q=\max _{1 \leq i \leq r} \frac{\left|P_{i}\right|}{\left|P_{0}\right|}, \quad M=\max \{1,(1+r) Q\} .
$$

When $\left|s^{\alpha}\right|>M$ and $\mathfrak{R} e(s) \geq 0$, the polynomial $\Delta(s, \tau)$ has the following estimates:

$$
\begin{aligned}
& |\Delta(s, \tau)| \\
& \geq\left|P_{0}\right|\left|\left(s^{\alpha}\right)^{r}\right|\left[1-\frac{\left|P_{1}\right|}{\left|P_{0}\right|} \frac{1}{\left|s^{\alpha}\right|}-\frac{\left|P_{2}\right|}{\left|P_{0}\right|} \frac{1}{\left|\left(s^{\alpha}\right)^{2}\right|}\right. \\
& \left.-\cdots-\frac{\left|P_{r}\right|}{\left|P_{0}\right|} \frac{1}{\left|\left(s^{\alpha}\right)^{r}\right|}\right] \\
& \geq M^{r} P_{0}\left[1-\frac{r Q}{(1+r) Q}\right]>0 .
\end{aligned}
$$

Thus, for $\left|s^{\alpha}\right|>M$ and $\mathfrak{R} e(s) \geq 0$, equation $\Delta(s, \tau)=0$ has no root.

In addition, from condition $\left(\mathrm{H}_{1}\right)$, we know that the roots of equation $\Delta(s, 0)=0$ are distributed in open left-half complex plane. When $\tau=0$ increases from zero, the roots of equation $\Delta(s, \tau)=0$ are distributed in right-half complex plane if and only if there exists some $s\left(|s| \leq M^{1 / \alpha}\right)$ such that $s$ passes through the imaginary axis. Yet, this is impossible by condition $\left(\mathrm{H}_{2}\right)$. Therefore, all the roots of equation $\Delta(s, \tau)=0$ lie in open left-half complex plane.

According to the proof of Theorem 9, we immediately have the following result.

Corollary 10. Assume that $(\bar{E}, \bar{A})$ is regular and $\operatorname{ind}(\bar{E}, \bar{A})=$ 1 ; then system (1) is delay-independently asymptotically stable if the following conditions simultaneously hold.

$\left(\mathrm{H}_{1}\right)^{\prime}$ All the roots of equation

$$
\Delta(s, 0)=\operatorname{det}\left[s^{\alpha} \bar{E}-\bar{A}-\bar{B}\right]=0
$$

have negative real parts.

$\left(\mathrm{H}_{2}\right)$ For any $y \in \mathbb{R}, \tau \in \mathbb{R}^{+}$and $i=\sqrt{-1}$, then

$$
\Delta(i y, \tau)=\operatorname{det}\left[(i y)^{\alpha} \bar{E}-\bar{A}-\bar{B} e^{-i y \tau}\right] \neq 0 .
$$


Note that condition $\left(\mathrm{H}_{2}\right)$ in Theorem 9 is a transcendental inequality, which is not convenient to use. Then we give an improved form as follows.

Theorem 11. Assume that $(\bar{E}, \bar{A})$ is regular and $\operatorname{ind}(\bar{E}, \bar{A})=1$; then system (1) is delay-independently asymptotically stable if the following conditions simultaneously hold.

$\left(\mathrm{H}_{3}\right)$ All the eigenvalues $\lambda$ s of matrix $\bar{A}_{1}+\bar{B}_{1}$ satisfy

$$
\left|\arg \left(\lambda\left(\bar{A}_{1}+\bar{B}_{1}\right)\right)\right|>\frac{\alpha \pi}{2} .
$$

$\left(\mathrm{H}_{4}\right)$ For any $y \in \mathbb{R}$, all the eigenvalues of the complex function matrix

$$
G(y)=\left[\bar{A}+\bar{B}-(i y)^{\alpha} \bar{E}\right]^{-1} \bar{B}-\frac{1}{2} I
$$

have negative real parts, that is, $\mathfrak{R} e[\lambda(G(y))]<0$.

Proof. From Theorem 9, we only need to prove condition $\left(\mathrm{H}_{4}\right)$ is equivalent to $\left(\mathrm{H}_{2}\right)$.

In fact, it follows from $\left(\mathrm{H}_{1}\right)$ and Corollary 10 that matrix $(\text { iy })^{\alpha} \bar{E}-\bar{A}-\bar{B}$ is nonsingular; then we have

$$
\begin{aligned}
\Delta(i y, \tau)= & \operatorname{det}\left[(i y)^{\alpha} \bar{E}-\bar{A}-\bar{B} e^{-i y \tau}\right] \\
= & \operatorname{det}\left[(i y)^{\alpha} \bar{E}-\bar{A}-\bar{B}\right] \\
& \times \operatorname{det}\left\{I-\left[(i y)^{\alpha} \bar{E}-\bar{A}-\bar{B}\right]^{-1} \bar{B}\left(e^{-i y \tau}-1\right)\right\} \\
& \neq 0 .
\end{aligned}
$$

Therefore,

$$
\operatorname{det}\left\{I-\left[(i y)^{\alpha} \bar{E}-\bar{A}-\bar{B}\right]^{-1} \bar{B}\left(e^{-i y \tau}-1\right)\right\} \neq 0 .
$$

Obviously, if $y \tau=2 k \pi(k=0, \pm 1, \pm 2, \ldots)$, then inequality (39) holds. Now, let $y \tau \neq 2 k \pi(k=0, \pm 1, \pm 2, \ldots)$; then we have

$$
\begin{aligned}
& \operatorname{det}\left[\frac{1}{e^{-i y \tau}-1} I-\left((i y)^{\alpha} \bar{E}-\bar{A}-\bar{B}\right)^{-1} \bar{B}\right] \neq 0, \\
& \quad \forall y \tau \neq 2 k \pi, k=0, \pm 1, \pm 2, \ldots, \quad y \in \mathbb{R}, \tau>0 .
\end{aligned}
$$

From inequality (40), we know that $\left(e^{-i y \tau}-1\right)^{-1}$ is not the eigenvalue of matrix $\left[(i y)^{\alpha} \bar{E}-\bar{A}-\bar{B}\right]^{-1} \bar{B}$, here $y \tau \neq 2 k \pi(\tau>$ $0, k=0, \pm 1, \pm 2, \ldots)$. In addition, it is easy to know

$$
\left[e^{-i y \tau}-1\right]^{-1}=-\frac{1}{2}+i \frac{\sin y \tau}{2(1-\cos y \tau)} .
$$

Then inequality (40) is equivalent to

$$
\mathfrak{R} e\left\{\lambda\left[\left((i y)^{\alpha} \bar{E}-\bar{A}-\bar{B}\right)^{-1} \bar{B}\right]\right\} \neq-\frac{1}{2}, \quad \forall y \in \mathbb{R} .
$$

When $y \rightarrow \infty$, we have

$$
\left|\lambda\left[\left((i y)^{\alpha} \bar{E}-\bar{A}-\bar{B}\right)^{-1} \bar{B}\right]\right| \longrightarrow 0,
$$

but

$$
\left|\left[e^{-i y \tau}-1\right]^{-1}\right| \geq \frac{1}{2} .
$$

Therefore, inequality (40) is equivalent to

$$
\mathfrak{R} e\left\{\lambda\left[\left((i y)^{\alpha} \bar{E}-\bar{A}-\bar{B}\right)^{-1} \bar{B}\right]\right\}>-\frac{1}{2}, \quad \forall y \in \mathbb{R} .
$$

Accordingly, we have

$$
\mathfrak{R} e\left\{\lambda\left[\left(\bar{A}+\bar{B}-(i y)^{\alpha} \bar{E}\right)^{-1} \bar{B}\right]\right\}<\frac{1}{2}, \quad \forall y \in \mathbb{R},
$$

which shows that condition $\left(\mathrm{H}_{2}\right)$ implies that condition $\left(\mathrm{H}_{4}\right)$ holds and vice versa. The proof is completed.

Next, we further consider the delay-independently asymptotic stability of system (2). The characteristic polynomial of system (2) can be represented as

$$
h(s, \tau)=\operatorname{det}\left[s^{\alpha} E-A-B e^{-s \tau}-s^{\alpha} C e^{-s \tau}\right] .
$$

Theorem 12. Assume that $(E, A)$ is regular and $\operatorname{ind}(E, A)=1$; then system (2) is delay-independently asymptotically stable if the following conditions simultaneously hold.

$\left(\mathrm{H}_{5}\right)$ All the roots of equation

$$
h(s, 0)=\operatorname{det}\left[s^{\alpha} E-A-B-s^{\alpha} C\right]=0
$$

have negative real parts.

$\left(\mathrm{H}_{6}\right)$ For any $y \in \mathbb{R}$ and $\tau \in \mathbb{R}^{+}$, then

$$
h(i y, \tau)=\operatorname{det}\left[(i y)^{\alpha} E-A-B e^{-i y \tau}-(i y)^{\alpha} C e^{-i y \tau}\right] \neq 0 .
$$

Proof. Let $\left.z(t)=D^{\alpha} x(t)\right)$; then system (2) can be written as

$$
\begin{gathered}
D^{\alpha} x(t)=z(t), \\
0=A x(t)-E z(t)+B x(t-\tau)+C z(t-\tau) .
\end{gathered}
$$

Take

$$
\begin{array}{ll}
\bar{x}(t)=\left[\begin{array}{l}
x(t) \\
z(t)
\end{array}\right], & \bar{E}=\left[\begin{array}{ll}
I & 0 \\
0 & 0
\end{array}\right], \\
\bar{A}=\left[\begin{array}{cc}
0 & I \\
A & -E
\end{array}\right], & \bar{B}=\left[\begin{array}{ll}
0 & 0 \\
B & C
\end{array}\right] ;
\end{array}
$$

then system (18) is equivalent to

$$
\bar{E} D^{\alpha} \bar{x}(t)=\bar{A} \bar{x}(t)+\bar{B} \bar{x}(t-\tau) .
$$


Hence, system (2) is delay-independently asymptotically stable if and only if system (52) is delay-independently asymptotically stable. Since

$$
\begin{array}{r}
\operatorname{det}\left[s^{\alpha} \bar{E}-\bar{A}-\bar{B}\right]=\operatorname{det}\left[\begin{array}{cc}
s^{\alpha} I & -I \\
-A-B & E-C
\end{array}\right] \\
=\operatorname{det}\left[s^{\alpha} E-A-B-\lambda C\right], \\
\operatorname{det}\left[(i y)^{\alpha} \bar{E}-\bar{A}-\bar{B} e^{-i y \tau}\right] \\
=\operatorname{det}\left[\begin{array}{cc}
(i y)^{\alpha} I & -I \\
-A-B e^{-i y \tau} & E-C e^{-i y \tau}
\end{array}\right] \\
=\operatorname{det}\left[(i y)^{\alpha} E-A-B e^{-i y \tau}-(i y)^{\alpha} C e^{-i y \tau}\right]
\end{array}
$$

are true, by Theorem 9 and Corollary 10, it is easy to get the conclusion. The proof is completed.

Similar to the analysis of Theorem 11, we have the following result.

Theorem 13. Assume that $(E, A)$ is regular and ind $(E, A)=1$; then system (2) is delay-independently asymptotically stable if the following conditions simultaneously hold.

$\left(\mathrm{H}_{7}\right)$ All the roots of equation

$$
h(s, 0)=\operatorname{det}\left[s^{\alpha} E-A-B-s^{\alpha} C\right]=0
$$

have negative real parts.

$\left(\mathrm{H}_{8}\right)$ For any $y \in \mathbb{R}$, all the eigenvalues of the complex function matrix

$$
H(y)=\left[A+B-(i y)^{\alpha}(E-C)\right]^{-1}\left[B+(i y)^{\alpha} C\right]-\frac{1}{2} I
$$

have negative real parts, that is, $\mathfrak{R} e[\lambda(H(y))]<0$.

Proof. We only need to prove that condition $\left(\mathrm{H}_{8}\right)$ is equivalent to $\left(\mathrm{H}_{6}\right)$. From condition $\left(\mathrm{H}_{5}\right)$, we know that the matrix $(i y)^{\alpha}(E-C)-A-B$ is nonsingular. Then, it follows from $\left(\mathrm{H}_{6}\right)$ that

$$
\begin{aligned}
h(i y, \tau)= & \operatorname{det}\left[(i y)^{\alpha} E-A-\left[B+(i y)^{\alpha} C\right] e^{-i y \tau}\right] \\
= & \operatorname{det}\left[(i y)^{\alpha}(E-C)-A-B\right] \\
& \times \operatorname{det}\left\{I-\left[(i y)^{\alpha}(E-C)-A-B\right]^{-1}\right. \\
& \left.\times\left[B+(i y)^{\alpha} C\right]\left(e^{-i y \tau}-1\right)\right\}
\end{aligned}
$$

$\neq 0$.

Then we obtain

$$
\begin{gathered}
\operatorname{det}\left\{I-\left[(i y)^{\alpha}(E-C)-A-B\right]^{-1}\left[B+(i y)^{\alpha} C\right]\left(e^{-i y \tau}-1\right)\right\} \\
\neq 0, \quad \forall y \tau \neq 2 k \pi, \quad k=0, \pm 1, \pm 2, \ldots, \quad y \in \mathbb{R}, \tau>0 .
\end{gathered}
$$

Obviously, if $y \tau=2 k \pi(k=0, \pm 1, \pm 2, \ldots)$, then inequality (58) holds. Now, let $y \tau \neq 2 k \pi(k=0, \pm 1, \pm 2, \ldots)$; then we have

$$
\begin{gathered}
\operatorname{det}\left\{\frac{1}{e^{-i y \tau}-1} I-\left[(i y)^{\alpha}(E-C)-A-B\right]^{-1}\left[B+(i y)^{\alpha} C\right]\right\} \\
\quad \neq 0, \quad \forall y \tau \neq 2 k \pi, \quad k=0, \pm 1, \pm 2, \ldots, \quad y \in \mathbb{R}, \tau>0 .
\end{gathered}
$$

From inequality (59), we know that $\left(e^{-i y \tau}-1\right)^{-1}$ is not the eigenvalue of matrix

$$
\left[(i y)^{\alpha}(E-C)-A-B\right]^{-1}\left[B+(i y)^{\alpha} C\right]
$$

where $y \tau \neq 2 k \pi(\tau>0, k=0, \pm 1, \pm 2, \ldots)$. In addition, it is easy to know

$$
\left[e^{-i y \tau}-1\right]^{-1}=-\frac{1}{2}+i \frac{\sin y \tau}{2(1-\cos y \tau)} .
$$

Then inequality (59) is equivalent to

$$
\mathfrak{R} e\left\{\lambda\left[\left[(i y)^{\alpha}(E-C)-A-B\right]^{-1}\left[B+(i y)^{\alpha} C\right]\right]\right\} \neq-\frac{1}{2} \text {. }
$$

When $y \rightarrow \infty$, we have

$$
\left|\lambda\left\{\left[(i y)^{\alpha}(E-C)-A-B\right]^{-1}\left[B+(i y)^{\alpha} C\right]\right\}\right| \rightarrow 0,
$$

but

$$
\left|\left[e^{-i y \tau}-1\right]^{-1}\right| \geq \frac{1}{2} .
$$

Therefore, inequality (59) is equivalent to

$$
\mathfrak{R} e\left\{\lambda\left[\left(A+B-(i y)^{\alpha}(E-C)\right)^{-1}\left(B+(i y)^{\alpha} C\right)\right]\right\}<\frac{1}{2},
$$

which shows that condition $\left(\mathrm{H}_{6}\right)$ implies that condition $\left(\mathrm{H}_{8}\right)$ is true and vice versa. The proof is completed.

Remark 14. Compared with Theorems 9 and 12, Theorems 11 and 13 actually offer two algebraic criteria of the delayindependently asymptotic stability of delayed fractionalorder linear singular differential systems, which is more concise and convenient to check the stability of such systems without solving the transcendental equations.

\section{An Illustrative Example}

The following example is presented to illustrate the usefulness of the proposed theoretical results.

Example 1. Consider two-dimensional Caputo fractionalorder singular differential difference system

$$
\begin{aligned}
& D^{1 / 3} x_{1}(t)=-x_{1}(t), \\
& x_{2}(t)=\frac{1}{2} x_{2}(t-\tau) .
\end{aligned}
$$


It can be written as

$$
\begin{gathered}
D^{1 / 3} x_{1}(t)=-x_{1}(t), \\
0=x_{2}(t)-\frac{1}{2} x_{2}(t-\tau) .
\end{gathered}
$$

Then we have

$$
\begin{gathered}
\bar{E}=\left[\begin{array}{ll}
1 & 0 \\
0 & 0
\end{array}\right], \quad \bar{A}=\left[\begin{array}{cc}
-1 & 0 \\
0 & 1
\end{array}\right], \\
\bar{B}=\left[\begin{array}{cc}
0 & 0 \\
0 & -\frac{1}{2}
\end{array}\right], \quad \bar{A}_{1}+\bar{B}_{1}=-2, \\
G(y)=\left[\bar{A}+\bar{B}-(i y)^{\alpha} \bar{E}\right]^{-1} \bar{B}-\frac{1}{2} I=\left[\begin{array}{cc}
-\frac{1}{2} & 0 \\
0 & -\frac{3}{2}
\end{array}\right] .
\end{gathered}
$$

It follows from equation $\operatorname{det}\left[\lambda I-\bar{A}_{1}-\bar{B}_{1}\right]=0$ that

$$
|\arg (\lambda)|=\pi>\frac{\alpha \pi}{2}
$$

Moreover, $\mathfrak{R} e[\lambda(G(y))]<0$. Thus, condition $\left(\mathrm{H}_{3}\right)$ and condition $\left(\mathrm{H}_{4}\right)$ are satisfied. From Theorem 11, we know that system (66) is delay-independently asymptotically stable.

In fact, when $\tau=0$, the solution of (50) is

$$
\begin{gathered}
x_{1}(t)=E_{\alpha}\left(-t^{\alpha}\right) x_{1}(0), \\
x_{2}(t)=0 .
\end{gathered}
$$

When $\tau \neq 0$, the solution of (50) is

$$
\begin{gathered}
x_{1}(t)=E_{\alpha}\left(-t^{\alpha}\right) x_{1}(0), \\
x_{2}(t)=\left(\frac{1}{2}\right)^{k} x_{2}(t-k \tau) .
\end{gathered}
$$

Here $t-k \tau \leq 0$, then $k \geq t / \tau$. When $t \rightarrow+\infty$, we have $k \rightarrow+\infty$. From [25], there exists a positive constant $M_{1} \geq 1$, such that for $\alpha=1 / 3 \in(0,1)$

$$
\left|E_{\alpha}\left(-t^{\alpha}\right)\right| \leq M_{1} e^{-t}
$$

When $t \rightarrow+\infty$, one can obtain

$$
x_{1}(t) \longrightarrow 0, \quad x_{2}(t) \longrightarrow 0 .
$$

Therefore, the zero solution of system (66) is asymptotically stable for any $\tau \in \mathbb{R}^{+}$; that is, system (66) is delayindependently asymptotically stable.

\section{Conclusions}

In this paper, the delay-independently asymptotic stability of delayed fractional-order linear singular differential systems has been discussed. We have synchronously taken into account the factors of such systems including Caputo fractional-order derivative, state delay, and singular coefficient matrices. In terms of the algebraic approach, some sufficient conditions are derived to ensure the asymptotic stability of the systems without solving the transcendental equations, which are very convenient to check the stability of such systems. An example is also provided to illustrate the theoretical results.

\section{Conflict of Interests}

The authors declare that there is no conflict of interests regarding the publication of this paper.

\section{Acknowledgments}

This work is supported by the National Natural Science Foundation of China under Grant nos. 11072059 and 61272530, the Specialized Research Fund for the Doctoral Program of Higher Education under Grant nos. 20110092110017 and 20130092110017, the Natural Science Foundation of Jiangsu Province of China under Grant no. BK2012741, the Programs of Educational Commission of Anhui Province of China under Grant nos. KJ2011A197 and KJ2013Z186.

\section{References}

[1] L. Dai, Singular Control Systems, vol. 118 of Lecture Notes in Control and Information Sciences, Springer, Heidelberg, Germany, 1989.

[2] P. Kunkel and V. Mehrmann, Differential-Algebraic Equations: Analysis and Numerical Solution, European Mathematical Society, Zürich, Switzerland, 2006.

[3] S. Xu, P. van Dooren, R. Ştefan, and J. Lam, "Robust stability and stabilization for singular systems with state delay and parameter uncertainty," IEEE Transactions on Automatic Control, vol. 47, no. 7, pp. 1122-1128, 2002.

[4] G. Lu and D. W. C. Ho, "Generalized quadratic stability for continuous-time singular systems with nonlinear perturbation," IEEE Transactions on Automatic Control, vol. 51, no. 5, pp. 818823, 2006.

[5] S. L. Campbell and V. H. Linh, "Stability criteria for differentialalgebraic equations with multiple delays and their numerical solutions," Applied Mathematics and Computation, vol. 208, no. 2, pp. 397-415, 2009.

[6] H. Wang, A. Xue, and R. Lu, "Absolute stability criteria for a class of nonlinear singular systems with time delay," Nonlinear Analysis, Theory, Methods \& Applications, vol. 70, no. 2, pp. 621630, 2009.

[7] J. Xu and J. Sun, "Finite-time stability of linear time-varying singular impulsive systems," IET Control Theory \& Applications, vol. 4, no. 10, pp. 2239-2244, 2010.

[8] Y. Ding, H. Zhu, S. Zhong, and Y. Zeng, "Exponential meansquare stability of time-delay singular systems with Markovian switching and nonlinear perturbations," Applied Mathematics and Computation, vol. 219, no. 4, pp. 2350-2359, 2012.

[9] I. Zamani, M. Shafiee, and A. Ibeas, "Exponential stability of hybrid switched nonlinear singular systems with time-varying delay," Journal of the Franklin Institute, vol. 350, no. 1, pp. 171193, 2013.

[10] T. Bergera and A. Ilchmanna, "On stability of time-varying linear differential-algebraic equations," International Journal of Control, vol. 86, no. 6, pp. 1060-1076, 2013. 
[11] K. S. Miller and B. Ross, An Introduction to the Fractional Calculus and Fractional Differential Equations, John Wiley \& Sons, New York, NY, USA, 1993.

[12] I. Podlubny, Fractional Differential Equations, vol. 198 of Mathematics in Science and Engineering, Technical University of Kosice, Kosice, Slovak Republic, 1999.

[13] A. A. Kilbas, H. M. Srivastava, and J. J. Trujillo, Theory and Applications of Fractional Differential Equations, vol. 204 of North-Holland Mathematics Studies, Elsevier Science, Amsterdam, The Netherlands, 2006.

[14] K. Diethelm, The Analysis of Fractional Differential Equations: An Application-Oriented Exposition Using Differential Operators of Caputo Type, Springer, Heidelberg, Germany, 2010.

[15] V. Lakshmikantham, S. Leela, and D. J. Vasundhara, Theory of Fractional Dynamic Systems, Cambridge Scientific, Cambridge, UK, 2009.

[16] D. Baleanu, J. A. T. Machado, and A. C. J. Luo, Fractional Dynamics and Control, Springer, 2012.

[17] V. Lakshmikantham and A. S. Vatsala, "Basic theory of fractional differential equations," Nonlinear Analysis: Theory, Methods \& Applications, vol. 69, no. 8, pp. 2677-2682, 2008.

[18] Y. Li, Y. Chen, and I. Podlubny, "Mittag-Leffler stability of fractional order nonlinear dynamic systems," Automatica, vol. 45, no. 8, pp. 1965-1969, 2009.

[19] Y. Li, Y. Chen, and I. Podlubny, "Stability of fractional-order nonlinear dynamic systems: lyapunov direct method and generalized Mittag-Leffler stability," Computers \& Mathematics with Applications, vol. 59, no. 5, pp. 1810-1821, 2010.

[20] J. Sabatier, M. Moze, and C. Farges, "LMI stability conditions for fractional order systems," Computers \& Mathematics with Applications, vol. 59, no. 5, pp. 1594-1609, 2010.

[21] X. Zhang, "Some results of linear fractional order time-delay system," Applied Mathematics and Computation, vol. 197, no. 1, pp. 407-411, 2008.

[22] M. P. Lazarević, "Finite time stability analysis of $\mathrm{PD}^{\alpha}$ fractional control of robotic time-delay systems," Mechanics Research Communications, vol. 33, no. 2, pp. 269-279, 2006.

[23] M. P. Lazarević and A. M. Spasić, "Finite-time stability analysis of fractional order time-delay systems: Gronwall's approach," Mathematical and Computer Modelling, vol. 49, no. 3-4, pp. 475481, 2009.

[24] W. Deng, C. Li, and J. Lü, "Stability analysis of linear fractional differential system with multiple time delays," Nonlinear Dynamics, vol. 48, no. 4, pp. 409-416, 2007.

[25] M. de la Sen, "About robust stability of Caputo linear fractional dynamic systems with time delays through fixed point theory," Fixed Point Theory and Applications, vol. 2011, Article ID 867932, 19 pages, 2011.

[26] C. P. Li and F. R. Zhang, "A survey on the stability of fractional differential equations," The European Physical Journal: Special Topics, vol. 193, no. 1, pp. 27-47, 2011.

[27] E. Kaslik and S. Sivasundaram, "Analytical and numerical methods for the stability analysis of linear fractional delay differential equations," Journal of Computational and Applied Mathematics, vol. 236, no. 16, pp. 4027-4041, 2012.

[28] S. J. Sadati, D. Baleanu, A. Ranjbar, R. Ghaderi, and T. Abdeljawad, "Mittag-Leffler stability theorem for fractional nonlinear systems with delay," Abstract and Applied Analysis, vol. 2010, Article ID 108651, 7 pages, 2010.

[29] D. Baleanu, S. J. Sadati, R. Ghaderi, A. Ranjbar, T. Abdeljawad, and F. Jarad, "Razumikhin stability theorem for fractional systems with delay," Abstract and Applied Analysis, vol. 2010, Article ID 124812, 9 pages, 2010.

[30] A. Mesbahi and M. Haeri, "Stability of linear time invariant fractional delay systems of retarded type in the space of delay parameters," Automatica, vol. 49, no. 5, pp. 1287-1294, 2013.

[31] I. N'Doye, M. Zasadzinski, M. Darouach, and N. Radhy, "Robust stabilization of uncertain descriptor fractional-order systems," Automatica, vol. 49, no. 6, pp. 1907-1913, 2013.

[32] Z. Zhang and W. Jiang, "Some results of the degenerate fractional differential system with delay," Computers \& Mathematics with Applications, vol. 62, no. 3, pp. 1284-1291, 2011. 


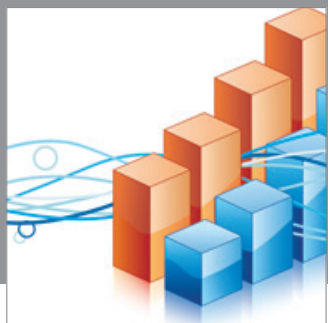

Advances in

Operations Research

mansans

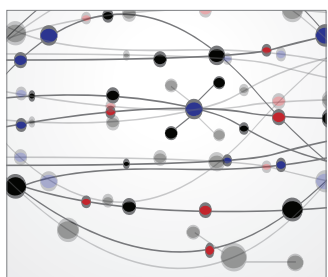

The Scientific World Journal
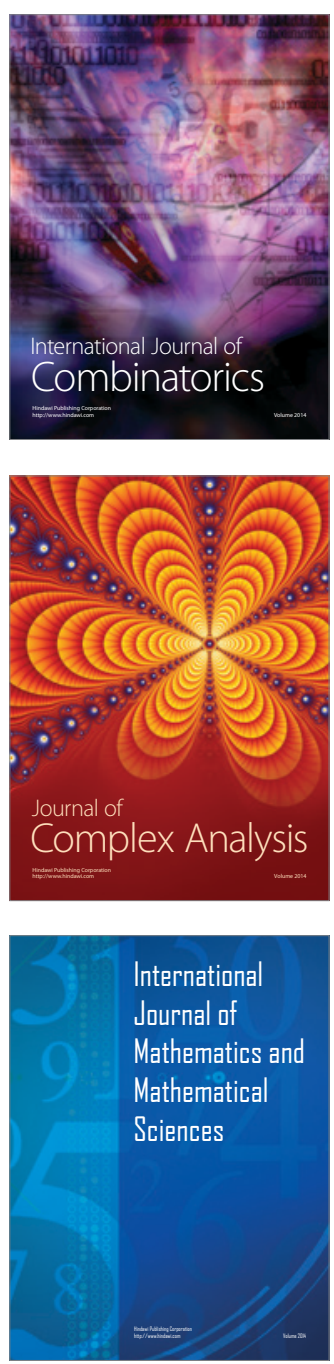
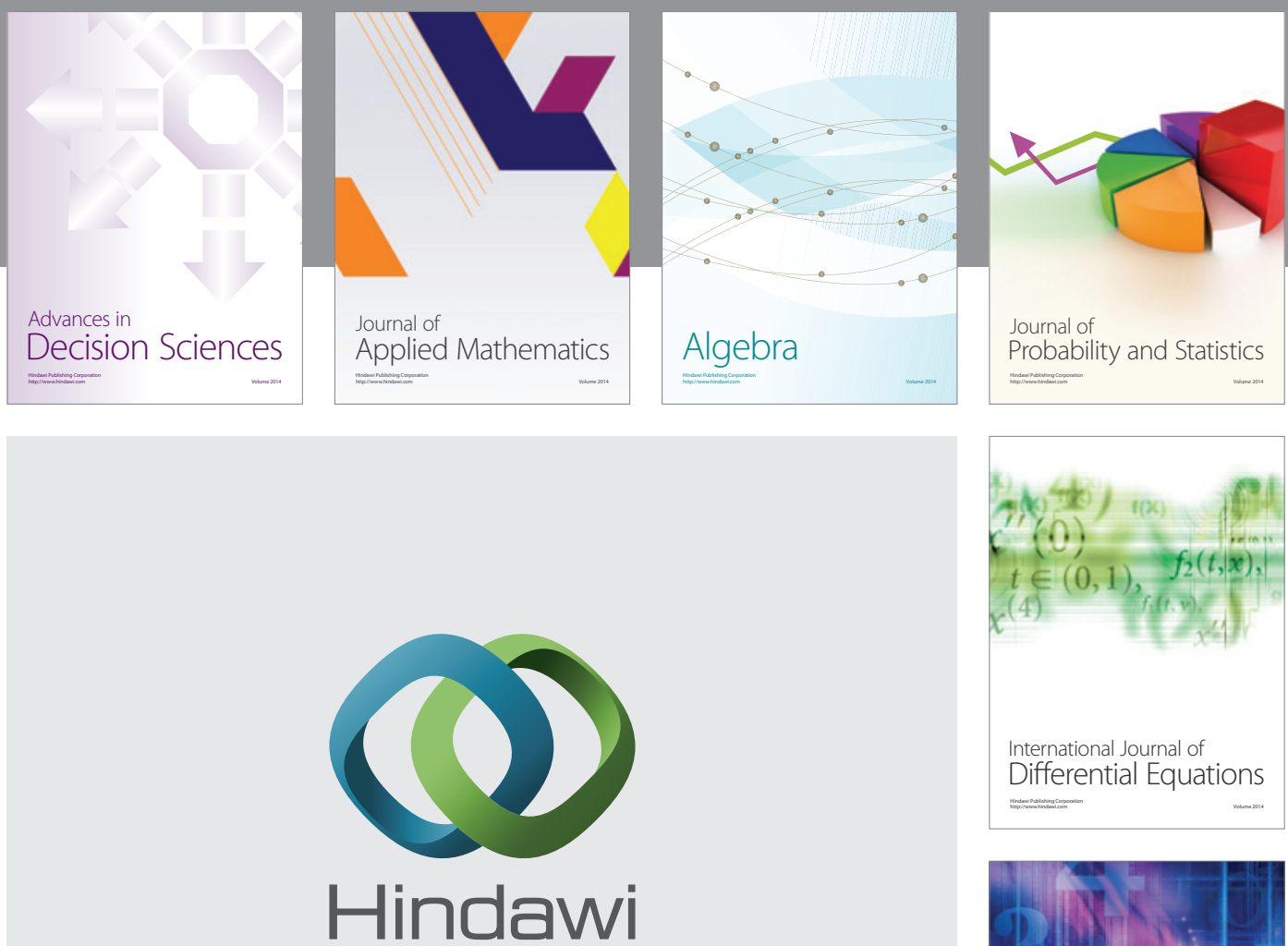

Submit your manuscripts at http://www.hindawi.com
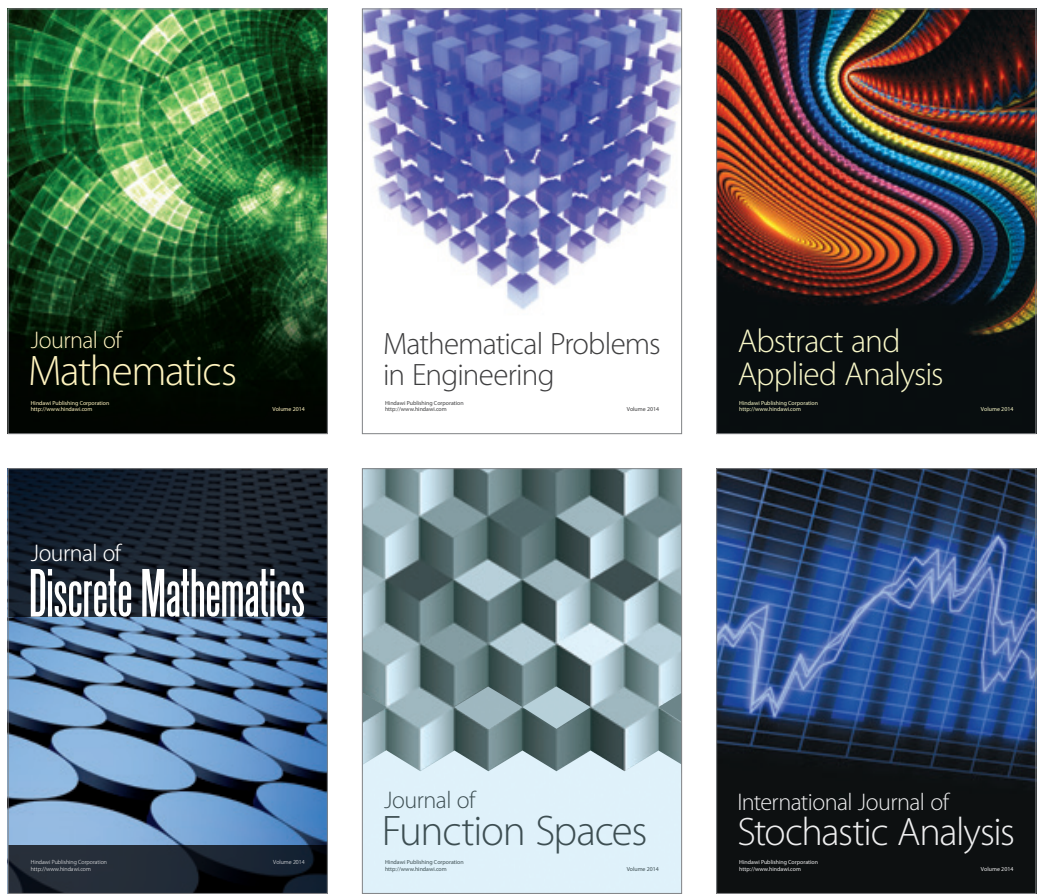

Journal of

Function Spaces

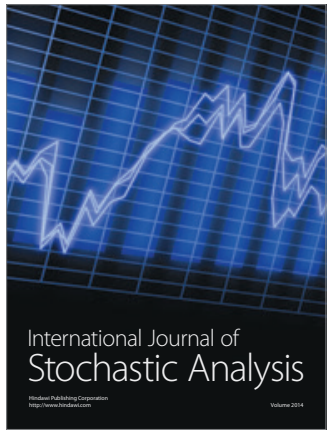

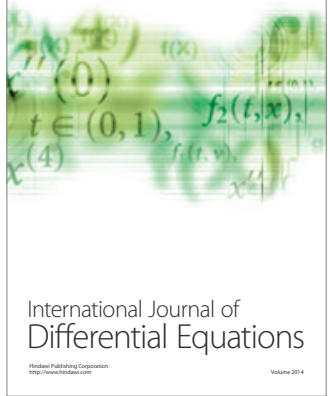
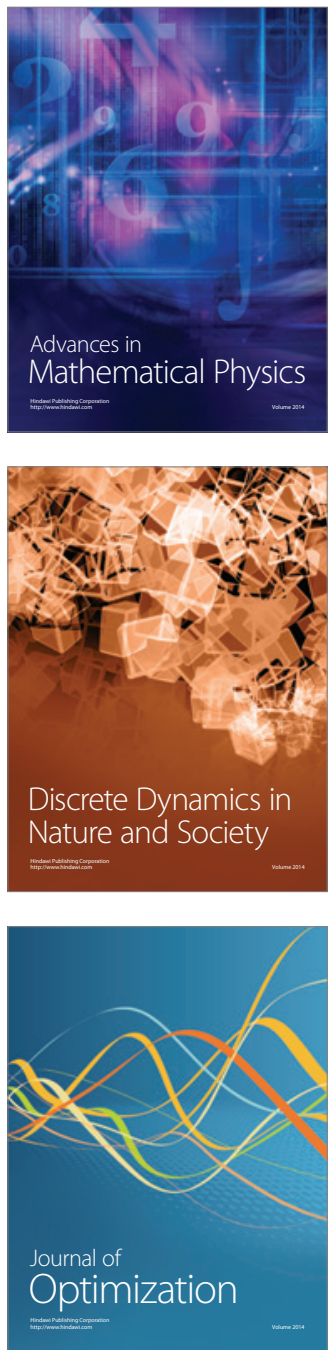